FLYCHK: generalized population kinetics and spectral model for rapid spectroscopic analysis for all elements

H.-K. Chung, M. Chen, W. L. Morgan, Y. Ralchenko, R. W. Lee

June 30, 2005

High Energy Density Physics 
This document was prepared as an account of work sponsored by an agency of the United States Government. Neither the United States Government nor the University of California nor any of their employees, makes any warranty, express or implied, or assumes any legal liability or responsibility for the accuracy, completeness, or usefulness of any information, apparatus, product, or process disclosed, or represents that its use would not infringe privately owned rights. Reference herein to any specific commercial product, process, or service by trade name, trademark, manufacturer, or otherwise, does not necessarily constitute or imply its endorsement, recommendation, or favoring by the United States Government or the University of California. The views and opinions of authors expressed herein do not necessarily state or reflect those of the United States Government or the University of California, and shall not be used for advertising or product endorsement purposes. 


\title{
FLYCHK: generalized population kinetics and spectral model for rapid spectroscopic analysis for all elements
}

\author{
H.-K. Chung ${ }^{1}$, M.H. Chen ${ }^{1}$, W.L. Morgan ${ }^{2}$, Yuri Ralchenko ${ }^{3}$ and R.W. Lee ${ }^{1}$ \\ ${ }^{1}$ Lawrence Livermore National Laboratory, L-399, PO Box 808, Livermore, CA 94551 \\ ${ }^{2}$ Kinema Research, PO Box 1147, Monument, CO 80132 \\ ${ }^{3}$ National Institute of Standards and Technology, Gaithersburg, MD 20899
}

\begin{abstract}
FLYCHK is a straightforward, rapid tool to provide ionization and population distributions of plasmas in zero dimension with accuracy sufficient for most initial estimates and in many cases applicable for more sophisticated analysis. FLYCHK solves rate equations for level population distributions by considering collisional and radiative atomic processes. The code is designed to be straightforward to use and yet is general enough to apply for most laboratory plasmas. Further, it can be applied for low-to-high Z ions and in either steady-state or time-dependent situations. Plasmas with arbitrary electron energy distributions, single or multiple electron temperatures can be studied as well as radiation-driven plasmas. To achieve this versatility and accuracy in a code that provides rapid response we employ schematic atomic structures, scaled hydrogenic crosssections and read-in tables. It also employs the jj configuration averaged atomic states
\end{abstract}


and oscillator strengths calculated using the Dirac-Hartree-Slater model for spectrum synthesis. Numerous experimental and calculational comparisons performed in recent years show that FLYCHK provides meaningful estimates of ionization distributions, well within a charge state for most laboratory applications.

Key Words: Collisional-radiative modeling, Hydrogenic model, Non-LTE kinetics, Plasma spectroscopy

Corresponding author, hchung@1lnl.gov, Fax. 925-422-0327, Tel. 925-423-3452. 


\section{Introduction}

There has been, and will continue to be, a resurgence in the development of novel plasma-generation techniques: x-ray lasers, ultra-short-pulse lasers, the National Ignition Facility (NIF), powerful z-pinch machines, VUV and X-ray Free Electron (XFEL) lasers. These developments indicate that there are expanding opportunities for the study of novel plasmas using the techniques associated with plasma spectroscopy. While the K-shell based spectroscopic code suite FLY and its predecessors [1] have been successfully employed to study hot dense plasmas for decades, the emphasis of the new plasma generation schemes are moving into uncharted regions of the temperature-density phase-space: warm dense matter, highly transient states of matter, and extremely hot and dense matter.

To provide a straightforward tool to assist experimental design and spectral analysis of both existing and future novel plasmas, we have developed a suite of codes consistent with the philosophy used to develop the older FLY suite of codes: simple, easy-to-use, fast, portable, and providing sufficiently reasonable spectroscopy for most applications [2]. The tool FLYCHK is general and can be applied to low-to-high Z ions under most conditions of laboratory plasmas: coronal, LTE or collisional-radiative plasmas, steady state or time-dependent cases, collision-drive or radiation-drive plasmas, Maxwellian or non-Maxwellian electron distributions, optically thin or thick plasmas with single species or mixture plasmas. It is compact enough to be used as a module for macroscopic codes such as radiation-hydrodynamics codes or PIC codes. Importantly, FLYCHK, unlike FLY, offers a first cut at ionization distributions for all Z that is useful 
for building more sophisticated kinetics models and can provide an easy-to-use method to study many critical elements of kinetic modeling.

The FLYCHK code suite employs schematic atomic structure and processes for population distributions. The built-in atomic sets are included for all ionization stages of atoms up to $Z=93$. Detailed population distributions are obtained by the solution of the rate equations considering collisional and radiative processes. For the detailed K-shell spectroscopy, the original FLY and HULLAC atomic data are implemented. Further, the

jj configuration atomic states and oscillator strengths from the Dirac-Hartree-Slater (DHS) model are employed for spectral synthesis in FLYSPEC. In this work we present a discussion of the FLYCHK suite development and examples relevant to several applications: laser-generated plasmas, EUV radiation modeling, EBIT plasmas, XFELgenerated plasmas.

\section{Population Kinetics Model}

A population kinetics model incorporates the results of atomic structure codes and scattering theories, plasma and statistical physics to describe atomic processes in atoms embedded in a plasma. The goal is to determine ionization and level population distributions of a plasma for a given electron temperature, $\mathrm{T}_{\mathrm{e}}$, and density, $\mathrm{N}_{\mathrm{e}}$, and then to assist in the analysis and prediction of spectroscopic observables. One way of achieving this goal is to solve rate equations for each energy level of each ion of an atom as it is influenced by the surrounding plasma; however, this requires a complete set of detailed atomic term energy levels, rates of atomic processes affecting the level population 
distribution and becomes computationally prohibitive. In practice, a kinetics model is usually constructed with a vastly reduced set of levels that can, as accurately as possible, represent the physical processes occurring in the plasma so that the spectroscopic observables are well represented. In this section, we present the elements of the population kinetics model implemented in FLYCHK.

\section{(1) Energy levels}

In order to keep the atomic data manageable, FLYCHK uses schematic atomic levels represented by their principal quantum number $\boldsymbol{n}$, an assumption that has been successfully applied for modeling ionization processes of plasmas [3,4]. Energies of the schematic levels are computed from ionization potentials. For all ground states we use the empirical [5] and calculated [6] ionization energies, which is critical for accurate ionization balance calculations. On the other hand, the ionization potential of an excited level with an outermost bound electron of the principal quantum number $\boldsymbol{n}$ is computed using the hydrogenic approximation with relativistic corrections;

$$
I_{n}=\frac{Q_{n}^{2} e^{2}}{n^{2} 2 a_{0}}\left(1+\left[\frac{\alpha Q_{n}}{n}\right]^{2}\left[\frac{2 n}{n+1}-\frac{3}{4}\right]\right)
$$

where $Q_{n}$ is the screened charge, $a_{0}$ Bohr radius and $e^{2} / 2 a_{0}$ is Rydberg energy. The screened charge is defined using a screening constant $\sigma(n, m)[3,4]$ as

$$
Q_{n}=Z_{n}-\sum_{m<n} \sigma(n, m) P_{m}-0.5 \sigma(n, n)\left(P_{n}-1\right)
$$

where $P_{n}$ is the occupation number of the level $\boldsymbol{n}$.

While bound levels are important for one-step ionization and recombination processes, in many cases, two-step processes of excitation-autoionization (EA) and 
dielectronic recombination (DR) play a pivotal role in ionization balance [7]. The effect of EA and DR channels on ionization balance is illustrated in Fig. 1 in a comparison with a measured xenon charge state distribution [8]. Shown in Fig. 1 are two implementations of the effect of autoionizing states. Autoionizing doubly-excited states are included as $\Delta \mathrm{n}=1$ dielectronic recombination (DR) channels which are critical in ionization balance for low $\mathrm{Z}$ atoms. The energy level of a doubly-excited state of an ion $\mathrm{X}^{(\mathrm{i})+}$, that is, $\mathrm{X}^{(\mathrm{i}+1)+}(\Delta \mathrm{n}=1$ excited state $)+\boldsymbol{n}$ is obtained as the energy difference between the ground state of an ion $\mathrm{X}^{\mathrm{i}+}$ and the excited state of the next ion $\mathrm{X}^{(\mathrm{i}+1)+}$ minus the ionization potential of the outmost electron $\boldsymbol{n}$ of the doubly-excited state. As an example, the energy of a doubly-excited state of $(1)^{2}(2)^{4}(3)^{1}(n)^{1}$ of O-like ion is given by the excited energy of $(1)^{2}(2)^{4}(3)^{1}$ of N-like ion and the ionization potentials (IP) of $\boldsymbol{n}$-shell electron and the ground state $(1)^{2}(2)^{6}$ of O-like ion as:

$$
\mathrm{E}\left[(\boldsymbol{1})^{2}(2)^{4}(3)^{1}(n)^{1}\right]-\mathrm{E}\left[(1)^{2}(2)^{6}\right]=\operatorname{IP}\left[(1)^{2}(2)^{6}\right]+\mathrm{E}\left[(1)^{2}(2)^{4}(3)^{1}\right]-\mathrm{E}\left[(1)^{2}(2)^{5}\right]-\mathrm{IP}[n]
$$

It is noted that the $\Delta \mathrm{n} \geq 2$ channels can be easily added; however, the occasionally important $\Delta \mathrm{n}=0$ channels [9] can not be included due to the assumption that the states of the same principal quantum number $\boldsymbol{n}$ and different angular momentum $\boldsymbol{l}$ are treated as one level.

In addition to the doubly-excited states described above, inner-shell excited states are critical in ionization balance for high $\mathrm{Z}$ atoms since their energy is close to the first ionization limit and hence EA and DR contributions through these states are substantial. The energy of the first inner-shell excited level with respect to the ground state of an ionization stage $\mathrm{X}^{\mathrm{i}+}$ is obtained as the difference between the ionization energy of the inner-shell electron of an ion $\mathrm{X}^{(\mathrm{i}-1)+}$ and that of the ground state of the ion $\mathrm{X}^{\mathrm{i}+}$. For 
example, the K-shell excited energy of O-like ion $(1)^{1}(2)^{7}$ is given by the ionization energy (IE) of K-shell electron and the ionization potential (IE) of the ground state $(1)^{2}(2)^{7}$ of F-like ion:

$$
\mathrm{E}\left[(1)^{1}(2)^{7}\right]-\mathrm{E}\left[(1)^{2}(2)^{6}\right]=\operatorname{IE}\left[(1) \text { of }(1)^{2}(2)^{7}\right]-\operatorname{IP}\left[(1)^{2}(2)^{7}\right] .
$$

The inner-shell excited levels with an $\boldsymbol{n}$-shell electron promoted from the first inner-shell excited level are sequentially constructed by adding the energy of bound $\boldsymbol{n}$ shell excited level with respect to its ground state to the energy of the first inner-shell excited level.

Although we have chosen a specific method for including the energies of the schematic levels there are many potential improvements that can be studied. For example, one method in consideration is to generate energy levels from more sophisticated atomic physics codes such as DHS [10] or HULLAC [11] for all ionization stages. Since the K-

shell spectroscopy widely-used in high energy density experiments requires fine-structure levels, FLYCHK utilizes the original FLY [1] and HULLAC [11] atomic data for Li- and He- and Hy-like ions to provide more accurate spectral synthesis. We are also investigating future possible models to include the angular-momentum $\boldsymbol{l}$-dependent states, which would allow an accounting of the $\Delta \mathrm{n}=0$ DR channels and provide a better representation of the ground configuration.

\section{(2) Radiative Processes}

For radiative transitions between bound states we include photo-excitation by absorption and deexcitation by spontaneous or stimulated emission of radiation field. An 
absorption oscillator strength of a transition from a level $n$ to a level $m$ was originally defined using the oscillator strength $\boldsymbol{f}_{\boldsymbol{H}}(\boldsymbol{n} \rightarrow \boldsymbol{m})$ of hydrogenic ions as $\boldsymbol{f}(\boldsymbol{n} \rightarrow \boldsymbol{m})=\boldsymbol{f}_{\boldsymbol{H}}(\boldsymbol{n} \rightarrow \boldsymbol{m}) \boldsymbol{P}_{\boldsymbol{n}}$, where $\mathbf{P}_{\mathrm{n}}$ is the occupation number of the level $\boldsymbol{n}$. We found, however, that $\boldsymbol{f}_{\boldsymbol{H}}(\boldsymbol{n} \rightarrow \boldsymbol{m})$ overestimates, by a factor of a few, the oscillator strength obtained by averaging those of the $\boldsymbol{l}$-dependent configurations belonging to the $\boldsymbol{n}$-shell and $\boldsymbol{m}$-shell. Here we use the averaged values derived from a relativistic Hartree-FockSlater atomic physics code [12]. The oscillator strength together with Einstein relations are used to generate the emission and absorption coefficient for bound-bound transitions. The spontaneous emission rate from a state $\boldsymbol{j}$ to a state $\boldsymbol{i}$ is directly related to the oscillator strength, $f_{i j}$ of the transition as

$$
A_{j i}=\frac{8 \pi^{2} e^{2} v_{j i}^{2}}{m c^{3}} \frac{g_{i}}{g_{j}} f_{i j}
$$

Here the $\boldsymbol{g}_{\boldsymbol{i}}$ and $\boldsymbol{g}_{\boldsymbol{j}}$ are the statistical weights of the states, $\boldsymbol{v}_{\boldsymbol{j} i}$ is the photon frequency, $\boldsymbol{e}$ and $\boldsymbol{m}$ are the electron charge and mass, and $\mathbf{c}$ is the speed of light. For the photoexcitation processes, the rate in units of $\mathrm{s}^{-1}$ is given by

$$
R_{i j}=4 \pi \int \alpha_{i j} J(v) \frac{d v}{h v} \text { where } J(v)=\frac{1}{2} \int_{-1}^{+1} I(v, \mu) d \mu
$$

where $\boldsymbol{J}(v)$ is the mean intensity, $I(v, \mu)$ is the specific intensity as a function of frequency $v$ and angle $\mu$, and the $\alpha_{i j}$ is the absorption cross-section for a transition from state $i$ to state $\boldsymbol{j}$. The absorption cross-section is $\alpha_{i j}=\frac{\pi e^{2}}{m c} f_{i j} \phi(v)$ in terms of the absorption oscillator strength of the transition $f_{i j}$, and the line profile function $\phi(v)$ where $\int_{-\infty}^{\infty} \phi(v) d v=1$. The photo de-excitation rate is related to the photo excitation rate through 
the Einstein relations that can be implemented by noting the emission and absorption oscillator strengths are related by $g_{i} f_{i j}=g_{j} f_{j i}$.

Photoionization processes are included to permit one to study the effects of the radiation field on the charge state distributions. The photoionization rate of ion $\mathrm{X}^{\mathrm{i}+}$ to $\mathrm{X}^{(\mathrm{i}+1)+}$ is given by

$$
R_{i i+1}=4 \pi \int \alpha_{i i+1} J(v) \frac{d v}{h v}
$$

We use the photoionization cross-section provided by Kramers' [13] and modified for the scaled hydrogenic approximation using the ionization potential of n-shell electron $I_{n}$ and Rydberg constant $I_{H}$

$$
\alpha_{i, i+1}(E)=\frac{64 \pi \alpha a_{0}^{2}}{3^{1.5}} \frac{I_{n}^{2.5} I_{H}^{0.5}}{Q_{n} E^{3}}=4.12 \times 10^{26} \frac{I_{n}^{5 / 2}}{Q_{n}} \frac{1}{v^{3}} ;
$$

here $\alpha$ is the fine structure constant and $a_{0}$ is the Bohr radius. The inverse process is the radiative recombination wherein a recombining electron emits the residual energy in radiation while no other electron plays a role. For Maxwellian distribution, the rate is given as

$$
R_{i+1 i}=\left(\frac{n_{i}}{n_{i+1}}\right)^{*} 4 \pi \int \alpha_{i i+1}\left(J(v)+\frac{2 h v^{3}}{c^{2}}\right) e^{-h v / k T_{e}} \frac{d v}{h v}
$$

The quantity $\left(\frac{n_{i}}{n_{i+1}}\right)^{*}$ represents the LTE ratio of the state of $X^{i+}$ to the state $X^{(i+1)+}$ given by the Saha-Boltzmann equation at $\mathrm{N}_{\mathrm{e}}$ and $\mathrm{T}_{\mathrm{e}}$, i.e.,

$$
\left(\frac{n_{i}}{n_{i+1}}\right)^{*}=1.66 \times 10^{-22} N_{e} \frac{g_{i}}{g_{i+1}} \frac{e^{U}}{T_{e}^{3 / 2}}
$$

where $U=I_{n} / T_{e}$. Note that the process above $R_{i+1 i}$ contains contributions from the both stimulated emission, the term $J(v))$ in the brackets, and spontaneous emission, the term 
$2 h v^{3} / c^{2}$. For an arbitrary electron distribution $f_{e}(E)$, an integration of the radiative recombination cross-section over the $f_{e}(E)$ is performed, such that

$$
R_{i+1, i}^{R R}=N_{e} \int_{0}^{\infty}\left(\frac{2 E}{m_{e}}\right)^{1 / 2} \sigma^{R R}(E) f_{e}(E) d E,
$$

where the cross-section $\sigma^{R R}$ has spontaneous and stimulated components $\sigma_{s p}$ and $\sigma_{s t}$ as

$$
\sigma^{R R}=\sigma_{s t}^{R R} J_{v}+\sigma_{s p}^{R R}=\left(J_{v}+\frac{2 h v^{3}}{c^{2}}\right) \sigma_{s t}^{R R} .
$$

The stimulated radiative recombination cross-section $\sigma_{s t}$ is written in terms of photoionization cross-section $\alpha_{\mathrm{u}+1}$ using the Einstein-Milne relation [14]

$$
\sigma_{s t}^{R R}(E)=\frac{g_{i}}{g_{i+1}} \frac{h^{2}}{4 m_{e} E} \frac{\alpha_{i i+1}(v)}{h v} .
$$

The radiation field can be specified by using either a Planckian radiation field at a radiation temperature $T_{r}$ or a data file. The Planckian radiation field at $T_{r}$ is given by

$$
J(v)=\frac{2 h v^{3}}{c^{2}} \frac{1}{e^{h \nu / k T_{r}}-1} .
$$

(3) Collisional processes

A collisional rate coefficient in units of $\mathrm{cm}^{3} \mathrm{~s}^{-1}$ is computed with the cross-section $\sigma(E)$ and a given electron energy distribution function $f_{e}(E)$ for a transition of threshold energy of $\boldsymbol{\Delta E}$ as

$$
R=N_{e} \int_{\Delta E}^{\infty} \mathrm{v} \sigma(E) f_{e}(E) d E,
$$


where $\mathbf{v}$ is the electron velocity at energy $\boldsymbol{E}$. For most applications, one may assume that the electron energy distribution function is the Maxwellian defined at a single temperature. Recently, however, there have been an increasing number of applications where this assumption breaks down and a more appropriate non-Maxwellian electron energy distribution function should be used to correctly represent collisional processes in a plasma.

We use a collisional excitation cross-section from a state $\boldsymbol{i}$ to a state $\boldsymbol{j}$ based on oscillator strength for the allowed transitions [15] as

$$
\sigma_{i j}(U)=\frac{8 \pi^{2} a_{0}^{2}}{\sqrt{3}}\left(\frac{I_{H}}{E_{i j}}\right)^{2} \frac{f_{i j} g(U)}{U},
$$

where $\boldsymbol{U}=\boldsymbol{E} / \boldsymbol{E}_{i j}, \boldsymbol{E}$ is the incoming electron energy, $\boldsymbol{E}_{i j}$ is the threshold energy of the transition, and $\boldsymbol{g}(\boldsymbol{U})$ is the effective gaunt factor. We use the gaunt factor suggested by Mewe [16], i.e., $g(U)=A+B U^{-1}+C U^{-2}+D \log U$ where $\boldsymbol{A}=0.15, \boldsymbol{B}=\boldsymbol{C}=0$ and $\boldsymbol{D}=0.28$. The rate coefficients are computed by integrating the cross-sections over the electron energy distribution, which in the case of a Maxwellian is

$$
C_{i j}\left(T_{e}\right)=\frac{R_{i j}\left(T_{e}\right)}{N_{e}}=1.578 \times 10^{-5} \frac{\bar{g}\left(E_{i j} / T_{e}\right) \exp \left(-E_{i j} / T_{e}\right)}{E_{i j} \sqrt{T_{e}}} f_{i j}
$$

where the averaged gaunt factor is given by $\bar{g}(y)=A+\left(B y-C y^{2}+D\right) e^{y} E_{1}(y)+C y$ and $\boldsymbol{E}_{i j}$ and $\boldsymbol{T}_{e}$ are in $\mathrm{eV}$.

The collisional de-excitation cross-section is obtained by the microscopic reversibility relation such that

$$
\sigma_{j i}(E)=\frac{g_{i}}{g_{j}} \frac{E+E_{i j}}{E} \sigma_{i j}\left(E+E_{i j}\right)
$$


For Maxwellian electron distribution, the de-excitation rate is also related to the excitation rate by the detailed balance such that, $n_{i}{ }^{*} C_{i j}=n_{j}{ }^{*} C_{j i}$ where the $n^{*}$ are the population densities in thermal equilibrium. The ratio $\left(\mathrm{n}_{\mathrm{i}} / \mathrm{n}_{\mathrm{j}}\right)^{*}$ of states in the same ion is given by the Boltzmann equation,

$$
\left(\frac{n_{i}}{n_{j}}\right)^{*}=\frac{g_{i}}{g_{j}} \exp \left[\frac{-\left(E_{i}-E_{j}\right)}{T_{e}}\right]=\frac{g_{i}}{g_{j}} \exp \left[\frac{E_{i j}}{T_{e}}\right] .
$$

For the Maxwellian case, one has the further option to use Coulomb-Born rate coefficients by Golden and Sampson [17].

There are a few schematic collisional ionization rates in FLYCHK. The simplest, and yet surprisingly successful, rates for most cases come from the work of Lotz [18,19]. The rate coefficient, for a Maxwellian, from an ion $\mathrm{X}^{\mathrm{i+}}$ to an ion $\mathrm{X}^{(\mathrm{i}+1)+}$ is given by

$$
\frac{R_{i, i+1}}{N_{e}}=2.97 \times 10^{-6}\left(\frac{\xi}{I_{n} \sqrt{T_{e}}}\right) E_{1}(U) .
$$

Here the $\xi$ is the number of electrons in the outer shell of the ion being ionized. The $\boldsymbol{E}_{\boldsymbol{l}}$ is the exponential integral of the first kind with the argument $\boldsymbol{U}$ where $\boldsymbol{U}=\boldsymbol{I}_{\boldsymbol{n}} / \boldsymbol{T}_{\boldsymbol{e}}$. A slightly modified semi-empirical formula of Burgess and Chidichimo [20] which may have a better representation for near-neutral ions and inner-shell ionization, can be used for collisional ionization from a level $\boldsymbol{n}$,

$$
\frac{R_{i, i+1}}{N_{e}}=2.715 \times 10^{-8} C \xi\left(\frac{I_{H}}{I_{n}}\right)^{3 / 2}\left(\frac{I_{n}}{T_{e}}\right)^{1 / 2} E_{1}\left(\frac{I_{n}}{T_{e}}\right) w\left(\frac{I_{n}}{T_{e}}\right),
$$


where $w\left(\frac{I_{n}}{T_{e}}\right)=\left[\log \left(1+\frac{\mathrm{T}_{\mathrm{e}}}{\mathrm{I}_{\mathrm{n}}}\right)\right]^{\frac{\beta}{1+T_{e} / I_{n}}}, \beta=0.25\left(\left[\frac{100 z+91}{4 z+3}\right]^{1 / 2}-5\right)$ and $\mathrm{z}$ is the charge of the ion. For the constant $\boldsymbol{C}$, the suggested value of 2 is used. The ionization cross-section is given by

$$
\sigma_{i i+1}(E)=\pi a_{0}^{2} C \xi\left[\frac{I_{H}}{I_{n}}\right]^{2}\left[\frac{I_{n}}{E}\right] \log \left[\frac{E}{I_{n}}\right] W\left[\frac{E}{I_{n}}\right]
$$

where for $\mathrm{E}>\mathrm{I}_{\mathrm{n}}, W\left[\frac{E}{I_{n}}\right]=\left[\log \left(\frac{E}{I_{n}}\right)\right]^{\frac{\beta I_{n}}{E}}$ and zero otherwise.

The third option is to use Coulomb-Born ionization rates [17] for the Maxwellian electron distribution cases. It was found that for the cases tested, the Coulomb-Born formula gave best overall agreement with HULLAC distorted-wave calculations, while Lotz and Burgess-Chidichimo rates agree within a factor of few. However, in some cases where the ionization stage had small population, the fit formula to Coulomb-Born rates can yield unphysical results. So, care in choosing this option is warranted.

For Maxwellian electron distribution, the three-body, or collisional recombination rate coefficient is related to the ionization rate coefficient by the detailed balance as

$$
R_{i+1, i}=1.66 \times 10^{-22} N_{e} \frac{g_{i}}{g_{i+1}} \frac{e^{U}}{T_{e}^{3 / 2}} R_{i, i+1}
$$

where $\boldsymbol{R}_{i, i+1}$ is the ionization rate coefficient. For arbitrary electron distributions, the rate coefficient is obtained by integrating the electron energy distribution over the differential ionization cross-section $\sigma_{i i+1}\left(E ; E^{\prime}, E^{\prime \prime}\right)$ which is the cross-section for the ionization of an atom from the ion $\mathrm{X}^{\mathrm{i}+}$ by an electron of energy $E$, resulting in an ejected and an outgoing electrons of energy $E^{\prime}$ and $E^{\prime \prime}$ and an ion $\mathrm{X}^{(\mathrm{i}+1)^{+}}$. This is related to the total ionization cross-section $\sigma_{i i+1}(E)$ through the relation [14,21], 


$$
\sigma_{i i+1}(E)=\iint \sigma_{i i+1}\left(E ; E^{\prime}, E^{\prime \prime}\right) d E^{\prime} d E^{\prime \prime}=\frac{1}{2} \int_{0}^{E-I_{n}} \sigma_{i i+1}\left(E, E_{b}\right) d E_{b}
$$

where $\boldsymbol{I}_{\boldsymbol{n}}$ is the threshold energy for the ionization process and $\boldsymbol{E}_{\boldsymbol{b}}$ is the energy of the ejected energy. By the microscopic reciprocity of differential cross-sections, the Fowler relation is given as [14]

$$
g_{i} E \sigma_{i i+1}\left(E ; E^{\prime} E^{\prime \prime}\right)=\frac{16 \pi m_{e}}{h^{3}} g_{i+1} E^{\prime} E^{\prime \prime} \sigma_{i+1 i}\left(E^{\prime} E^{\prime \prime} ; E\right)
$$

The three-body recombination rate coefficient is given as

$$
\begin{gathered}
R=N_{e}^{2} \iiint\left(\frac{2 E^{\prime}}{m_{e}}\right)^{1 / 2}\left(\frac{2 E^{\prime \prime}}{m_{e}}\right)^{1 / 2} f_{e}\left(E^{\prime}\right) f_{e}\left(E^{\prime \prime}\right) \sigma_{i+1 i}\left(E^{\prime} E^{\prime \prime} ; E\right) d E^{\prime} d E^{\prime \prime} d E \\
=N_{e}^{2} \frac{g_{i}}{2 g_{i+1}} \frac{h^{3}}{2^{5 / 2} \pi m_{e}^{3 / 2}} \iiint\left(\frac{2 E}{m_{e}}\right)^{1 / 2}\left(\frac{E}{E^{\prime} E^{\prime \prime}}\right)^{1 / 2} f_{e}\left(E^{\prime}\right) f_{e}\left(E^{\prime \prime}\right) \sigma_{i i+1}\left(E ; E^{\prime} E^{\prime \prime}\right) d E d E^{\prime} d E^{\prime \prime} .
\end{gathered}
$$

We currently use the simple assumption that the differential ionization cross-section $\sigma_{i i+1}\left(E ; E^{\prime}, E^{\prime \prime}\right)$ is a given incoming energy $E$, which can be replaced by more accurate descriptions in the future.

(4) Autoionization and electron capture

FLYCHK has two sets of autoionizing states, inner-shell excited and doublyexcited states described above. For autoionization rates, we make two assumptions to obtain schematic rates [22] : (1) An autoionization rate from an autoionizing state $\boldsymbol{k}$ of an ion $\mathrm{X}^{\mathrm{i}+}$ (consisting of the excited state $\boldsymbol{j}$ of the next ion $\mathrm{X}^{(\mathrm{i}+1)+}$ plus the outer most excited electron) to a bound state $\boldsymbol{i}$ of the ion $\mathrm{X}^{(\mathrm{i}+1)^{+}}$is obtained from detailed balancing of the corresponding electron capture cross-section from the state $\boldsymbol{i}$ to $\boldsymbol{k}$ of the ion $\mathrm{X}^{(\mathrm{i}+1)+}$. The electron capture cross-section is approximated by the collisional excitation cross- 
section from the state $\boldsymbol{i}$ to the excited state $\boldsymbol{j}$ of the ion $\mathrm{X}^{(\mathrm{i}+1)+}$ at the threshold energy $\boldsymbol{E}_{\boldsymbol{i} j}$. Note that the bound state $\boldsymbol{j}$ of the ion $\mathrm{X}^{(\mathrm{i}+1)+}$ is the continuum limit of the autoionizing state $\boldsymbol{k}$ of the ion $\mathrm{X}^{\mathrm{i}+}$. Using the Saha equation and a Maxwellian distribution $\boldsymbol{f}_{e}{ }^{M}$, the relation between the electron capture cross-section $\sigma_{\mathfrak{c}}$ and autoionization rate $\boldsymbol{A}$ is written:

$$
\frac{N_{e} \int \sigma_{c} v f_{e}^{M}\left(E_{i j}\right) d E}{A}=\frac{g_{k}}{g_{i}} \frac{1}{2}\left[\frac{2 \pi \hbar^{2}}{m T_{e}}\right] \exp \left[-\frac{E_{i j}}{T_{e}}\right]
$$

where $f_{e}^{M}(E)=\frac{2}{\sqrt{\pi}} \frac{E^{1 / 2}}{T_{e}^{3 / 2}} \exp \left[-\frac{E}{T_{e}}\right]$.

Assuming that the resonance occurs with the width of

$$
\frac{d E}{d n}=\frac{d}{d n}\left(\frac{Z^{2} R y}{n^{2}}\right)=-\frac{2 Z^{2} R y}{n^{3}},
$$

we obtain the following relation:

$$
\frac{\sigma_{c}\left(E_{i j}\right)}{A}=\frac{g_{k}}{g_{i}} \frac{\pi^{2} n^{3} \hbar a_{0}^{2}}{4 E_{i j} Z^{2}}
$$

Then using the Van Regemorter cross-section [15] and a gaunt factor at the threshold energy $\boldsymbol{E}_{i j}$ for $\boldsymbol{\sigma}_{\mathfrak{c}}\left(\boldsymbol{E}_{i j}\right)$, we obtain the autoionization rate as

$$
A=\frac{32}{\sqrt{3}} \frac{Z^{2}}{\hbar n^{3}} \frac{g^{i}}{g^{k}} \frac{I_{H}^{2}}{E_{i j}} f_{i j} g\left(E_{i j}\right)=5.835 \times 10^{17} \frac{Z^{2}}{n^{3}} \frac{g^{i}}{g^{k}} \frac{f_{i j}}{E_{i j}}
$$

We have performed a study of the form by comparing this rate with values calculated by Dirac-Hartree-Slater (DHS) code [23] and found that the gaunt factor 0.15 gives a better agreement than the standard value 0.12 .

(5) Plasma effects 
In addition to the ionization and excitation processes, plasma electric fields can effectively reduce the ionization potential of an ion and hence affect the charge state distributions of a plasma. The electron and ion fields compete with the atomic field particularly for the higher-lying $\mathbf{n}$-shell states and for certain states the orbital electrons may not be bound $[24,25]$ For sufficiently low density plasmas, the Debye length determines the limit of the bound states and for dense plasmas, the volume per ion determines which orbits can be considered bound states. We apply the formulation of ionic potential depression (IPD) model of Stewart and Pyatt [25] to determine those states that are above the depressed ionization potential and these are excluded from the rate equations.

Currently the highest Rydberg state of any ion is set to the principal quantum number of 10 for computational efficiency. This assumption is based on the facts that the higher-lying states are either depressed out by IPD or are in LTE with their continuum states. In the latter case the rates into and out of a state near its continuum limit are essentially equivalent so the state can be removed from the rate matrix. This assumption can be readily tested out in FLYCHK by increasing the maximum principal quantum number. As an example, the IPD cut-off occurs at $\boldsymbol{n}=150$ for a krypton plasma at $\mathrm{T}_{\mathrm{e}}=3.5$ $\mathrm{keV}$ and $\mathrm{N}_{\mathrm{e}}=10^{18} \mathrm{~cm}^{-3}$. We performed FLYCHK calculations using $\boldsymbol{n}_{\boldsymbol{m a x}}=5,10$ and 150 and 450. By explicitly calculating the population distribution, we found that the Rydberg states with $\boldsymbol{n}>20$ are in LTE with their continuum limit and the differences in charge state distributions are negligible for cases using $\boldsymbol{n}_{\max }=10,150$ and 450 but are of significance 
for $\boldsymbol{n}=5$. For $\mathrm{N}_{\mathrm{e}}=10^{10} \mathrm{~cm}^{-3}$, the LTE state occurs near 450 and yet the charge state distributions are comparable for all three cases of $\boldsymbol{n}_{\max }=10,150$ and 450.

\section{Spectral Modeling: FLYSPEC}

The post-processor FLYSPEC synthesizes the emission and absorption spectra using the population distributions from the FLYCHK output file. However, the spectrum using level populations of the $\boldsymbol{n}$-based configurations from FLYCHK will in many cases fail to represent observable spectra due to the simplicity of the atomic structure and radiative transitions employed in the $\boldsymbol{n}$-based data file. Therefore, to improve on this we generate atomic data of relativistic $\mathrm{jj}$ configuration and oscillator strength using DHS atomic physics code [10] and then use this more detailed atomic data to statistically redistribute the FLYCHK level population among the jj configuration populations deriving a more satisfying spectral representation.

To generate the spectrum, the emissivity, $\boldsymbol{\varepsilon}_{\boldsymbol{v}}$, and opacity, $\boldsymbol{\kappa}_{\boldsymbol{v}}$, of the plasma as a function of frequency, or equivalently, energy are calculated [26]. In keeping with the spirit of FLYCHK it is assumed that the plasma is a slab with uniform temperature and density giving rise to the emitted intensity

$$
I_{v}=S_{v}\left(1-e^{-\tau}\right) \quad\left(\mathrm{ergs} / \mathrm{cm}^{2} / \mathrm{sec} / \mathrm{Hz} / \Omega\right)
$$

where $\boldsymbol{S}_{v}$ is the source function and $\tau_{v}$ is the optical depth. These are defined as

$$
S_{v}=\varepsilon v / \kappa v, \quad\left(\mathrm{ergs} / \mathrm{cm}^{2} / \mathrm{sec} / \mathrm{Hz} / \Omega\right) \text { and } \tau_{v}=\kappa v L
$$

where $\boldsymbol{L}$ is the geometric length of the slab. 
The emissivity and opacity are determined for all transitions, both bound-bound and bound-free by recourse to the populations in the file generated by FLYCHK. The emissivity for a bound-bound transition between the upper level ' $u$ ' and the lower level ' $l$ ' is given by

$$
\boldsymbol{\varepsilon}_{\boldsymbol{v}}=N_{u} A_{u l} h \boldsymbol{v}_{u l} / 4 \pi \phi(\boldsymbol{V}) \quad\left(\mathrm{ergs} / \mathrm{cm}^{3} / \mathrm{sec} / \mathrm{Hz} / \Omega\right)
$$

where the population density of the state $\boldsymbol{u}$ is $\boldsymbol{N}_{\boldsymbol{u} \boldsymbol{l}}$ and $\boldsymbol{v}_{\boldsymbol{u} \boldsymbol{l}}$ is the frequency of the transition. The line profile function $\phi(v)$ determines the frequency dependence of the emissivity. The opacity of the same transition is given by

$$
\kappa v=N_{l}\left(1-N_{u} g_{l} / N_{l} g_{u}\right) \pi e^{2} / m c f_{l u} \phi(v)\left(\mathrm{cm}^{-1}\right)
$$

These two formulae completely specify the source function for the bound-bound transitions.

For bound-free emissivity, we use hydrogenic cross-section. That is, the edge has a sharp onset at the ionization potential and slowly decays as $v^{-3}$. The formula for the emissivity from a state in ion stage $\boldsymbol{i}+\boldsymbol{1}$ recombining to a state in ion stage $\boldsymbol{i}$ is

$$
\varepsilon_{v}=1.01 \times 10^{-42}\left(N_{i+1} N_{e} \frac{g_{i}}{g_{i+1}} \frac{I_{p}^{5 / 2}}{T_{e}^{3 / 2} Z} e^{h\left(v_{I P}-v\right) / k T_{e}}\right) G
$$

where the $\boldsymbol{I}_{\boldsymbol{p}}$ and the $\boldsymbol{v}_{\boldsymbol{I} \boldsymbol{p}}$ are the ionization potential of the state $\boldsymbol{i}$ in $\mathrm{eV}$ and $\mathrm{Hz}$, respectively. The $G$ is the Gaunt factor from the calculations of Karzas and Latter [27]. The opacity of the same transition is

$$
\kappa_{v}=4.13 \times 10^{26} \frac{N_{i} I_{p}^{5 / 2}}{Z v^{3}}\left(1-1.66 \times 10^{-22} \frac{n_{i+1} g_{i}}{n_{i} g_{i+1}} \frac{N_{e}}{T_{e}^{3 / 2}} e^{h\left(v_{I P}-v\right) / k T_{e}}\right) G .
$$

Note that the second term in the brackets is the correction to the bound-free absorption due to stimulated recombination and is usually small. 
The emissivity due to the transitions of the free electrons between continuum states interacting with a collection of ions of population densities $\boldsymbol{n}_{\boldsymbol{i}}$ and charges $\boldsymbol{z}_{\boldsymbol{i}}$ is

$$
\varepsilon_{v}=5.05 \times 10^{-41} \frac{N_{e}}{T_{e}^{1 / 2}} \sum N_{i} z_{i}^{2} e^{-h v / k T_{e}},
$$

where the sum is taken over all ionization stages and there is no threshold for the process. The opacity for the free-free process is

$$
\kappa_{v}=3.43 \times 10^{6} \frac{N_{e}}{T_{e}^{1 / 2}} \sum N_{i} z_{i}^{2} \frac{1}{v^{3}}\left(1-e^{-h v / k T_{e}}\right) .
$$

\section{Results}

We show an example of FLYCHK and FLYSPEC calculations compared with spectroscopically determined charge state distributions and measured spectra of a xenon gas jet experiment by Chenais-Popovics et al. [8]. The xenon plasma is assumed to be steady-state and uniform, $\mathrm{T}_{\mathrm{e}}$ is determined by Thomson scattering measurement to be in the range of $415-455 \mathrm{eV}$ and the ion density is $4.75 \times 10^{18} \mathrm{~cm}^{-3}$. Figure 2 shows the experimentally determined charge state distribution compared to those of two FLYCHK calculations, the first curve (A) uses the Van Regemorter's collisional excitation rates and the second curve (B) uses those of Sampson. This illustrates that FLYCHK can predict average charge states and spectra within a charge state, which is quite reasonable for the initial designing of experiments. Further, the variation of the collision rates does not change this conclusion. Figure 3 shows the two synthetic xenon spectra for the two cases compared with the measured spectra of ref. [8]. Though neither of spectra reproduces the measured spectrum precisely, it is promising that this simple code can predict the position 
and intensity of such complex spectra in a reasonable accuracy, which again lends credence to the statement that FLYCHK can be used as an initial experimental design tool.

FLYCHK has been developed for a wide range of applications in plasma experiments. An application of current interest is the study of time-dependent ionization processes in ultra-short-pulse laser-produced plasmas where non-thermal, energetic electrons are present, in addition to cold electrons in the near-solid density matter. The example we study is the irradiation of a solid $\mathrm{Cu}$ sample by a laser at $10^{18} \mathrm{~W} / \mathrm{cm}^{3}$ creating a non-thermal "hot" electron distribution with a temperature of $200 \mathrm{keV}$ and density of $10^{20} \mathrm{~cm}^{-3}$ that generate $\mathrm{K}$-shell vacancies in the relatively cooler solid density $\mathrm{Cu}$. We then study the time-dependence of the ionization as a function of the temperature of the warm $\mathrm{Cu}$. Figure. 4 shows the mean ionization as a function of time for the three plasma temperatures of $10,100,1000 \mathrm{eV}$. This indicates the time needed for the plasma to arrive at a steady state for a given condition of $\mathrm{T}_{e}$ and $\mathrm{N}_{\mathrm{e}}$, and provides an insight on whether the shifts and widths of K- $\alpha$ lines, which are generated by the hot electrons, can be used as a time-independent diagnostic for given plasma conditions. As the hot electrons will require many collisions before completely thermalizing with the bulk electrons, Figure. 4 indicates that the solid density $\mathrm{Cu}$ ions come to a steady state, i.e., the curves flatten, by 100 fs. The higher the temperature, the longer it took to reach the steady state. Roughly speaking, this supports the idea that the shifts and widths of K- $\alpha$ lines reflect the steadystate charge state distributions for a given $\mathrm{T}_{\mathrm{e}}$ and provide a thermal electron diagnostic. For reference we include in Fig. 4 the mean charge one would obtain by performing the FLYCHK calculations at the matter temperature for both the NLTE steady-state case and 
the LTE case - note we displace the two along the time axis for ease of observation. Normally LTE state occurs when collisions dominate radiative rates. Since collisional deexcitation and recombination rates are more dominant over radiative rates as $\mathrm{T}_{\mathrm{e}}$ gets lower and ions are less charged, the lower $\mathrm{T}_{\mathrm{e}}$ cases have higher chance of coming to an equilibrium for a given $\mathrm{N}_{\mathrm{e}}$. We can observe that for the 10 and $100 \mathrm{eV}$ cases the NLTE steady state comes very close to LTE while for the $1000 \mathrm{eV}$ case LTE is not achieved.

For long-pulse laser-produced plasmas, such as gas bag or hohlraum experiments [28], K-shell spectroscopy and FLY have been widely used so the application of FLYCHK is straightforward. While the steady-state assumption is often valid for these long-pulse plasmas, in many cases one has to include the effect of opacity in both kinetics and spectrum calculations, both of which are incorporated into FLYCHK. Recently, laser-irradiated under-dense foams have been explored as a potential test bed for nonLTE kinetics simulations as these are relatively uniform and can reach steady-state conditions [28]. In Fig. 5 we show a comparison of FLYCHK results at $\mathrm{T}_{\mathrm{e}}=500 \mathrm{eV}$ and $\mathrm{N}_{\mathrm{i}}=6 \times 10^{20} \mathrm{~cm}^{-3}$ with an absolute intensity measurement from an under-dense Ti-doped $\mathrm{SiO}_{2}$ foam experiments. This illustrates that one can use FLYCHK as a spectroscopic diagnostic tool to study a plasma consisting of mixtures.

Beyond laser-created plasmas, FLYCHK can be applied to a variety of physical situations such as photoionizing plasmas created by Z-pinch plasmas [29] and warm dense matter created by proton-heating or EUV-emitting plasmas for industrial applications. Figure 6 shows the charge state distributions of Sn plasmas for conditions that tend to maximize the EUV emission, which is of interest for lithography. Indeed, the results in Fig. 6 compare well with the literature in the EUV lithography community [30]. 
Comparisons with numerous other simulations and experiments suggest that the accuracy of FLYCHK increases with electron density. Figure 7 shows the charge state distributions of Au at $T_{e}=2.5 \mathrm{keV}$ and $\mathrm{N}_{\mathrm{e}}=10^{12} \mathrm{~cm}^{-3}$ of the FLYCHK calculation compared to an EBIT experiment [31]. The measured average $\mathrm{Z}$ is $46.8 \pm 0.8$ while FLYCHK gives $Z=48.5$. For higher electron densities, FLYCHK is in better agreement with the measurements. Indeed, published Au data from foil, with $\mathrm{N}_{\mathrm{e}}=6 \times 10^{20}, \mathrm{~T}_{\mathrm{e}}=2.2$ $\mathrm{keV}$, and $\left\langle\mathrm{Z}>=49.3 \pm 0.5\right.$ [32], and hohlraum [33] experiments with $\mathrm{N}_{\mathrm{e}}=1.4 \times 10^{21} \mathrm{~cm}^{-3}$, $\mathrm{T}_{\mathrm{e}}=2.6 \mathrm{keV}$ and $\langle\mathrm{Z}\rangle=51$, are in better agreement with FLYCHK results of 49.6 and 50.4, respectively. We speculate that this trend can be attributed to the atomic structure of using $\boldsymbol{n}$ configuration as the basic level in FLYCHK. When fine structure level populations are not distributed according to their statistical weights, FLYCHK will overestimate collisional mixing among these states since the more detailed level populations are assumed to be statistically distributed. Therefore, the error associated with this assumption will increase as the plasma density decreases. This is where resonant processes by electron capture or excitation may lead to highly non-LTE distributions amongst those states.

\section{Summary and Future work}

The FLYCHK suite of codes is a tool for studying a variety of plasma spectroscopy for a wide variety of sources providing populations, ionization distributions and spectra. It is simple and easy to use, while generating reasonable estimates. Finally, we are also developing a Boltzmann solver that will be integrated with FLYCHK suite to study 
electron energy distributions self-consistently with the population distributions of interest for a wide range of non-thermal plasma, e.g, XFEL, intense short pulse laser, and beam generated plasmas.

\section{Acknowledgement}

This work was performed under the auspices of the U.S. Department of Energy by University of California, Lawrence Livermore Laboratory under Contract W-7405-Eng48. Authors would like to thank fellow collaborators who have motivated and helped developing FLYCHK and, in particular, those who participated in the $3^{\text {rd }}$ Non-LTE Kinetics workshop.

\section{References}

[1] Lee RW, Larsen JT. A time-dependent model for plasma spectroscopy of K-shell emitters, J Quant Spectrosc Radiat Transfer. 1996;56:535

[2] Chung HK, Morgan WL, Lee RW. FLYCHK: an extenstion to the K-shell spectroscopy kinetics model FLY. J Quant Spectrosc Radiat Transfer. 2003;81:107

[3] Lee YT. A model for ionization balance and L-shell spectroscopy of non-LTE plasmas. J Quant Spectrosc Radiat Transfer. 1987;38:131

[4] Marchand R, Caille S, Lee YT. Improved screening coefficients for the hydrogenic ion model. J. Quant Spectrosc Radiat Transfer. 1990;43:149

[5] Kelly RL, Journal of Physical and Chemical Reference Data, Supplement. 1987;16:1 
[6] Scofield JH. Energies Of Hydrogen- and Helium-Like Ions For Z From 6 To 54.

Internal Document, UCID-16848, Lawrence Livermore National Laboratory, 1975

Saloman EB, Hubbell JH, Scofield JH, X-ray attenuation cross sections for energies 100 $\mathrm{eV}$ to $100 \mathrm{keV}$ and elements $\mathrm{Z}=1$ to $\mathrm{Z}=92$. Atomic Data \& Nuclear Data Tables, 1988:38:1

[7] Lee RW, Nash JK, Ralchenko Y, Review of the NLTE kinetics code workshop, J. Quant Spectrosc Radiat Transfer. 1997;58:737

[8] Chenais-Popovics C, Malka V, Gauthier JC, Gary S, Peyrusse O, Rabec-Le Gloahec M, Matsushima I, Bauche-Arnoult C, Bachelier A, Bauche J. X-ray emission of a xenon gas jet plasma diagnosed with Thomson scattering Phys. Rev. E 2002;65:046418 [9] Savin DW, Bartsch T, Chen MH, Kahn SM,. Liedahl DA, Linkemann J, Müller A, Schippers S, Schmitt M, Schwalm D, and Wolf A, Dielectronic Recombination in Photoionized Gas: The Importance of Fine-structure Core Excitations. Ap. J. 1997;489:L115

[10] Huang KN, Aoyagi M, Chen MH, Crasemann B. Neutral-atom electron binding energies from relaxed-orbital relativistic Hartree-Fock-Slater calculations. At. Dat. Nuclear. Dat. Tables 1976;18:243

[11] Bar-Shalom A, Klapisch M, Oreg J. HULLAC, an integrated computer package for atomic processes in plasmas. J. Quant Spectrosc Radiat Transfer. 2001;71:169

[12] Scofield JH. Phys. Rev. A. 1989;40:3054

[13] Kramers H. Philos Mag. 1923:46:836

[14] Oxenius J. Kinetic Theory of Particles and Photons, Berlin; Springer-Verlag, 1986. p. $21-65$ 
[15] Van Regemorter H. Ap. J. 1962;136:906

[16] Mewe R. Interpolation formulae for the electron impact excitation of ions in the H-, He-, Li, and Ne-sequences. Astron. Astrophys. 1972;20:215

[17] Golden LB, Sampson DH, Electron-impact cross-sections and rates for $\mathrm{nl} \rightarrow \mathrm{n}$ 'l' transitions in hydrogenic ions and hydrogen. Astrophys. J. 1971;170:181

[18] Lotz W, Z. Phys. 1968;216:241

[19] Lotz W, Z. Phys. 1969;220:266

[20] Burgess A, Chidichimo MC. Electron impact ionization of complex ions. Mon. Not. R. astr. Soc. 1983;203:1269

[21] Shoub EC. Departures of the electron energy distribution from a Maxwellian in hydrogen. I. Formulation and solution of the electron kinetic equation. Ap J. Suppl. Ser. $1977 ; 34: 259$

[22] Sobelman II, Vainshtein LA, Yukov EA. Excitation of atoms and broadening of spectra lines. $2^{\text {nd }}$ Ed. Berlin; Springer, 1995. p. 120-124

[23] Chen MH, Laiman E, Casemann B, Aoyagi M, Mark H. Relativistic L-shell Auger and Coster-Kronig rates and fluorescence yields. Phys. Rev. A. 1979;19;2253

[24] Inglis DR, Teller E. Ionic depression of series limits in one-electron spectra.

Astrophys. J. 1939;90:439

[25] Stewart JC, Pyatt KD. Lowering of ionization potentials in plasmas. Astrophys. J. $1966 ; 144: 1203$

[26] Mihalas D. Stellar Atmospheres, $2^{\text {nd }}$ Ed. San Francisco; W.H. Freeman, 1978. Ch. 4 [27] Karzas WJ, Latter R. Electron radiative transitions in a coulomb field. Ap. J. Suppl. $1961 ; 55: 167$ 
[28] Back CA, Feldman U, Weaver JL, Seely J, Constantin C, Holland G, Lee RW, Chung HK, Scott HA, Absolute, Time-resolved Emission of Non-LTE L-shell Spectra from Ti-doped Aerogels in preparation

[29] Foord ME, Heeter RF, van Hoof PAM, Thoe RS, Bailey JE, Cuneo ME, Chung HK, Liedahl DA, Fournier KB, Chandler GA, Jonauskas V, Kisielius R, Mix LP, Ramsbottom C, Springer PT, Keenan FP, Rose SJ, Goldstein WH. Charge-State distribution and Doppler effect in an expanding photoionized plasma. Phys Rev Lett 2004;93:055002

[30] Colombant D, Tonon GF. X-ray emission in laser-produced plasmas. J Appl Phys 1973;444:3524; Choi IW, Daido H, Yamagami S, Nagai K, Norimatsu T, Takabe H, Suzuki M, Nakayama T, Matsui T. Detailed space-resolved characterization of a laserplasma soft-x-ray source at 13.5 -nm wavelength with tin and its oxides. JOSA B. $2000 ; 17: 1616$

[31] Wong KL, May MJ, Beiersdorfer P, Fournier KB, Wilson B, Brown GV, Springer P. Determination of the charge state distribution of a highly ionized coronal Au plasma. Phys. Rev. Lett 2003;90:235001

[32] Foord ME, Glenzer SH, Thoe RS, Wong KL, Fournier KB, Wilson BG, Springer PT, Ionization processes and charge-state distribution in a highly ionized high-Z laserproduced plasmas, Phys. Rev. Lett 2000;85:992

[33] Glenzer SH, Fournier KB, Wilson BG, Lee RW, Suter LJ, Ionization balance in inertial confinement fusion hohlraums, Phys Rev Lett 2001;87:045002 
Figure Captions

Fig. 1. Calculated average charge states of Xe ions are plotted as a function of $\mathrm{T}_{\mathrm{e}}$ for various dielectronic recombination (DR) models in FLYCHK. The inclusion of dielectronic recombination (DR) channels with inner-shell (IS) processes can be seen to play an important role in charge state distributions of high $\mathrm{Z}$ ions. Burgess-Mertz formula are known to overestimate the DR contribution for high- $\mathrm{Z}$ ions by a factor of few. The experimental measurement of Ref. [8] is indicated by the *.

Fig. 2. Charge state distributions of Xe ions are compared for measured values [8] and FLYCHK results at $\mathrm{T}_{\mathrm{e}}=415 \mathrm{eV}$ and $\mathrm{N}_{\mathrm{i}}=4.75 \times 10^{18} \mathrm{~cm}^{-3}$. (A) uses he Van Regemorter collisional excitation rates and (B) Sampson rates. Both results agree with measurement within $\sim 1$ charge state.

Fig. 3. Synthetic spectra for the two cases of (A) and (B) of Fig. 2 are compared with the measured spectra of Ref. [8].

Fig. 4. Time-dependent calculations of average charge states of $\mathrm{Cu}$ ions as a function of time for 3 different bulk $\mathrm{T}_{\mathrm{e}}$ cases, i.e., $10 \mathrm{eV}, 100 \mathrm{eV}$ and $1 \mathrm{keV}$. Ions are assumed to be at solid density and $\mathrm{N}_{\mathrm{e}}$ is self-consistently computed by charge neutrality. A non-thermal hot electron component at $200 \mathrm{keV}$ having a density of $0.1 \% \mathrm{~N}_{\mathrm{e}}$ are also included in the simulations. Note that the time-dependent values approach to the steady-state (circles) and LTE (squares) values within 100 fs. 
Fig. 5. Synthetic spectra of Si, $\mathrm{O}$ and Ti ions at $\mathrm{T}_{\mathrm{e}}=500 \mathrm{eV}$ and $\mathrm{N}_{\mathrm{i}}=6 \times 10^{20} \mathrm{~cm}^{-3}$ are compared with measured spectra of Ti-doped $\mathrm{SiO}_{2}$ under-dense foam [28]. The experimental intensity is an absolute measurement.

Fig. 6. Steady-state charge state distributions of $\mathrm{Sn}$ ions are plotted for $\mathrm{T}_{\mathrm{e}}=28 \mathrm{eV}, 32 \mathrm{eV}$ and $36 \mathrm{eV}$. These results compare very well with Ref. [30].

Fig. 7. Gold charge state distributions are compared between FLYCHK and EBIT measurement [31] at $\mathrm{T}_{\mathrm{e}}=2.5 \mathrm{keV}$ and $\mathrm{N}_{\mathrm{e}}=10^{12} \mathrm{~cm}^{-3}$. 


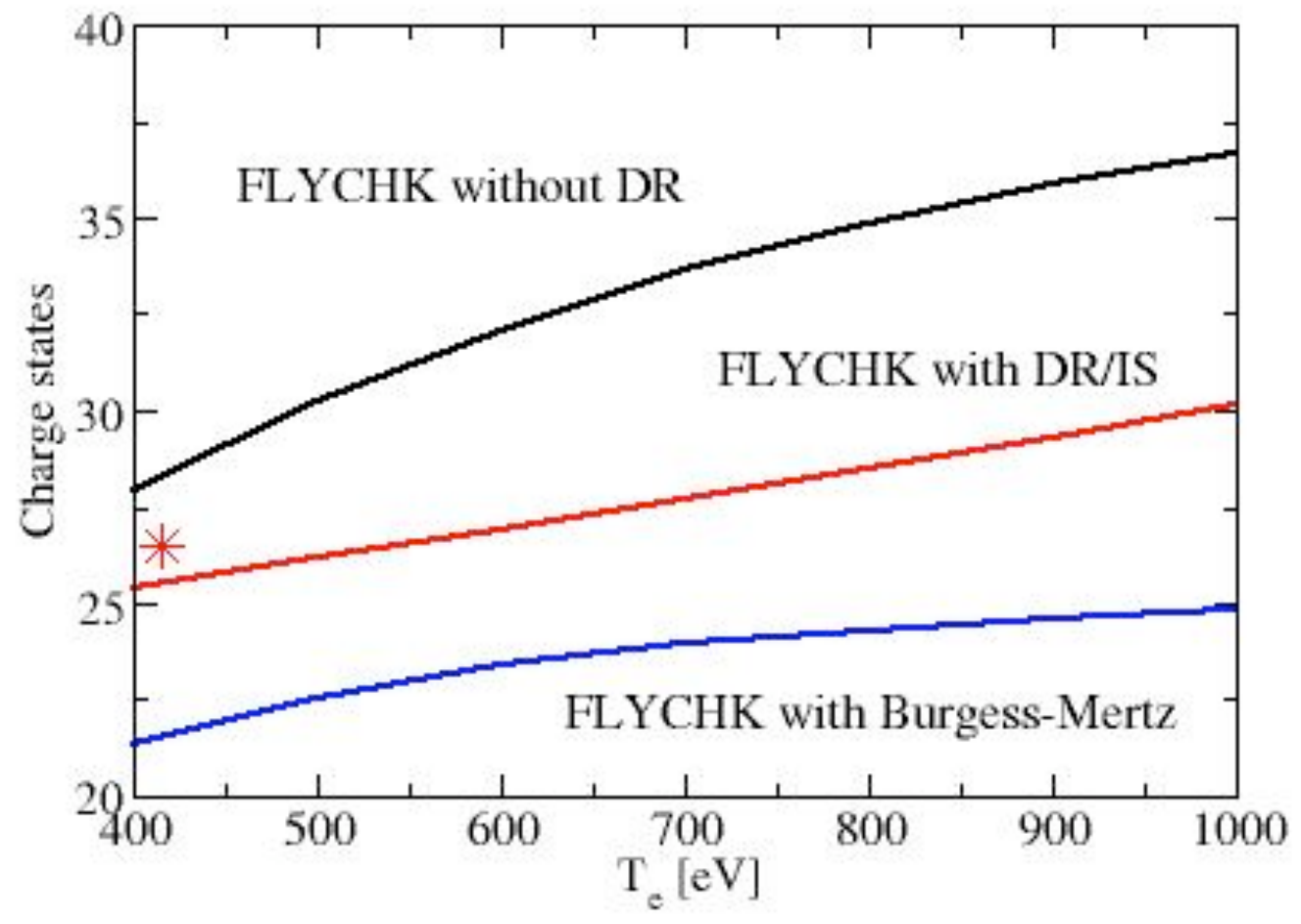




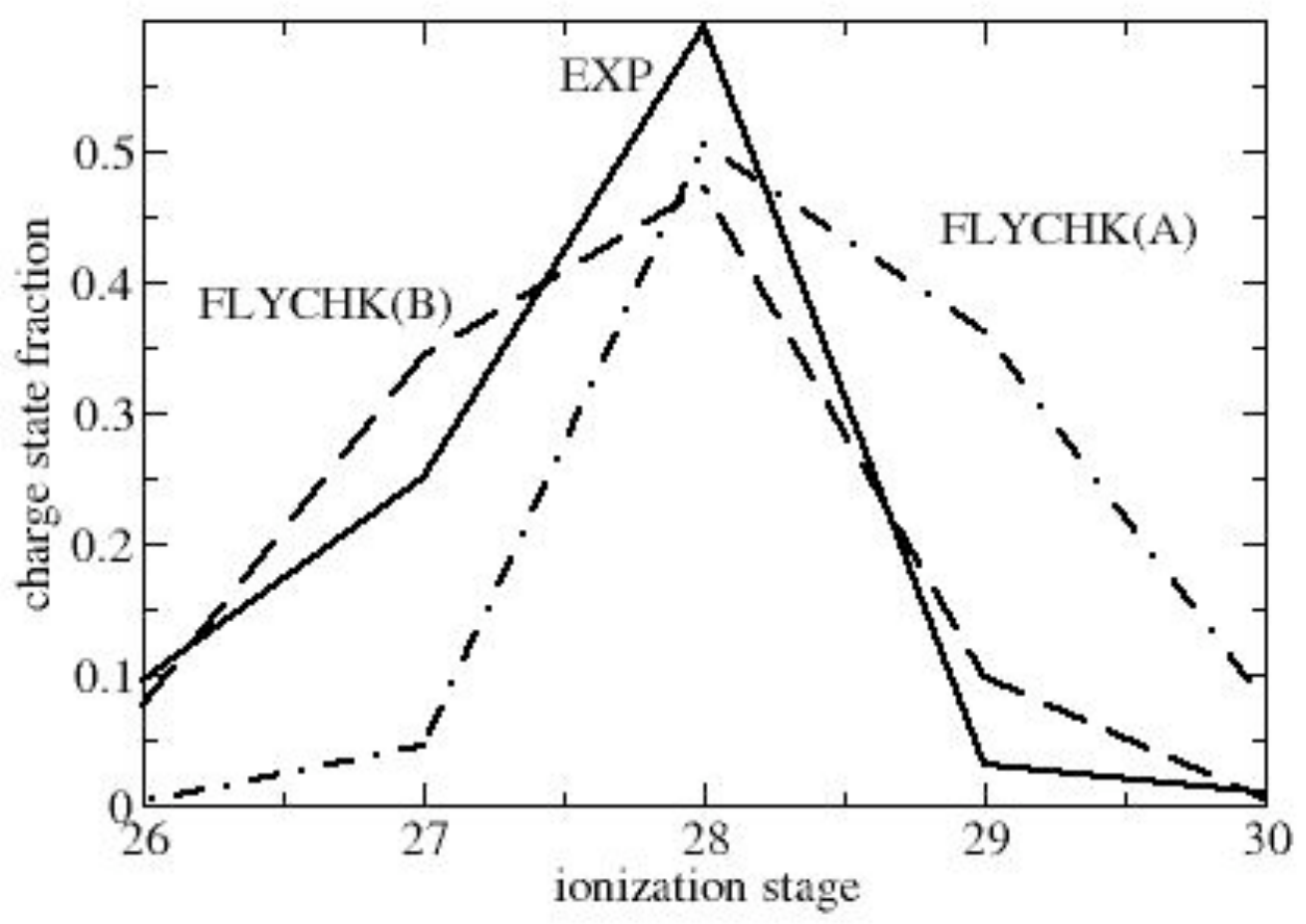




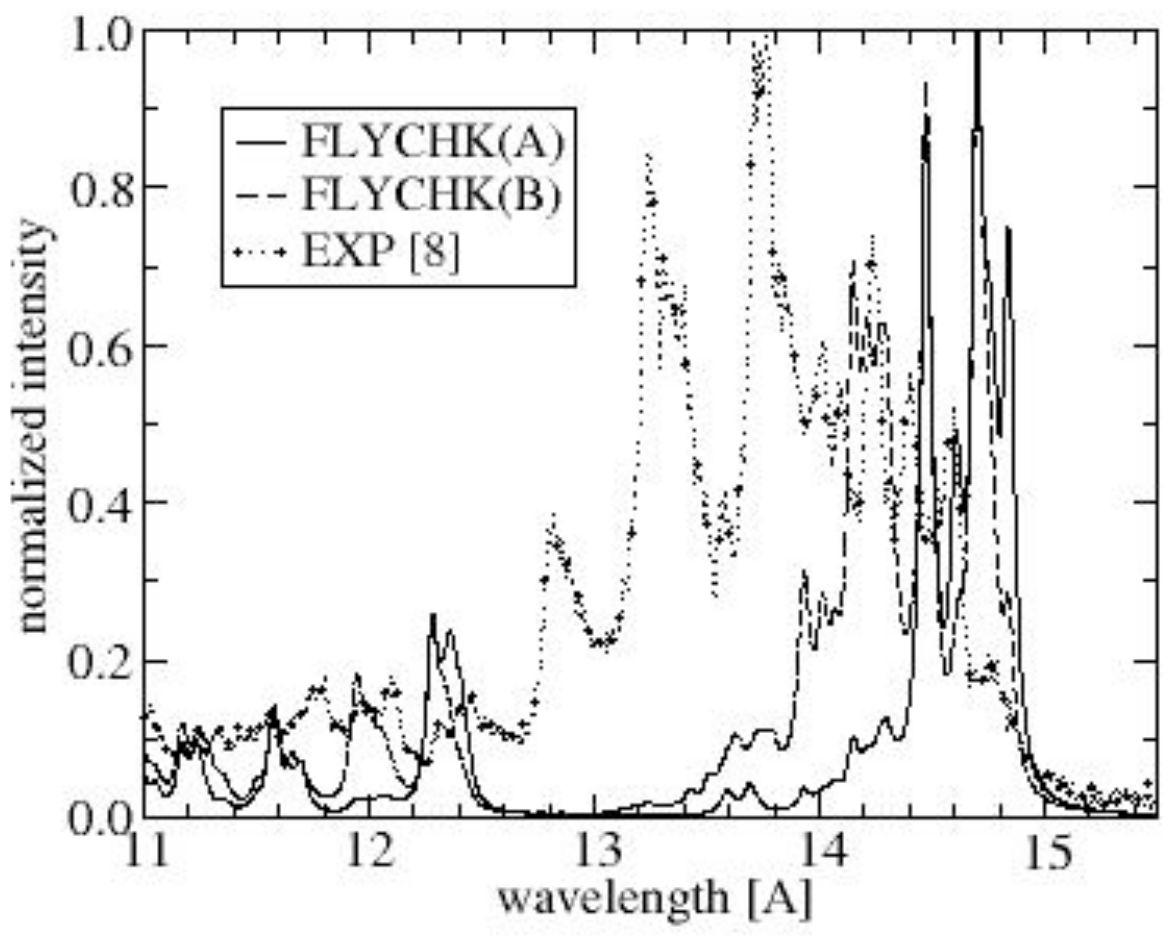




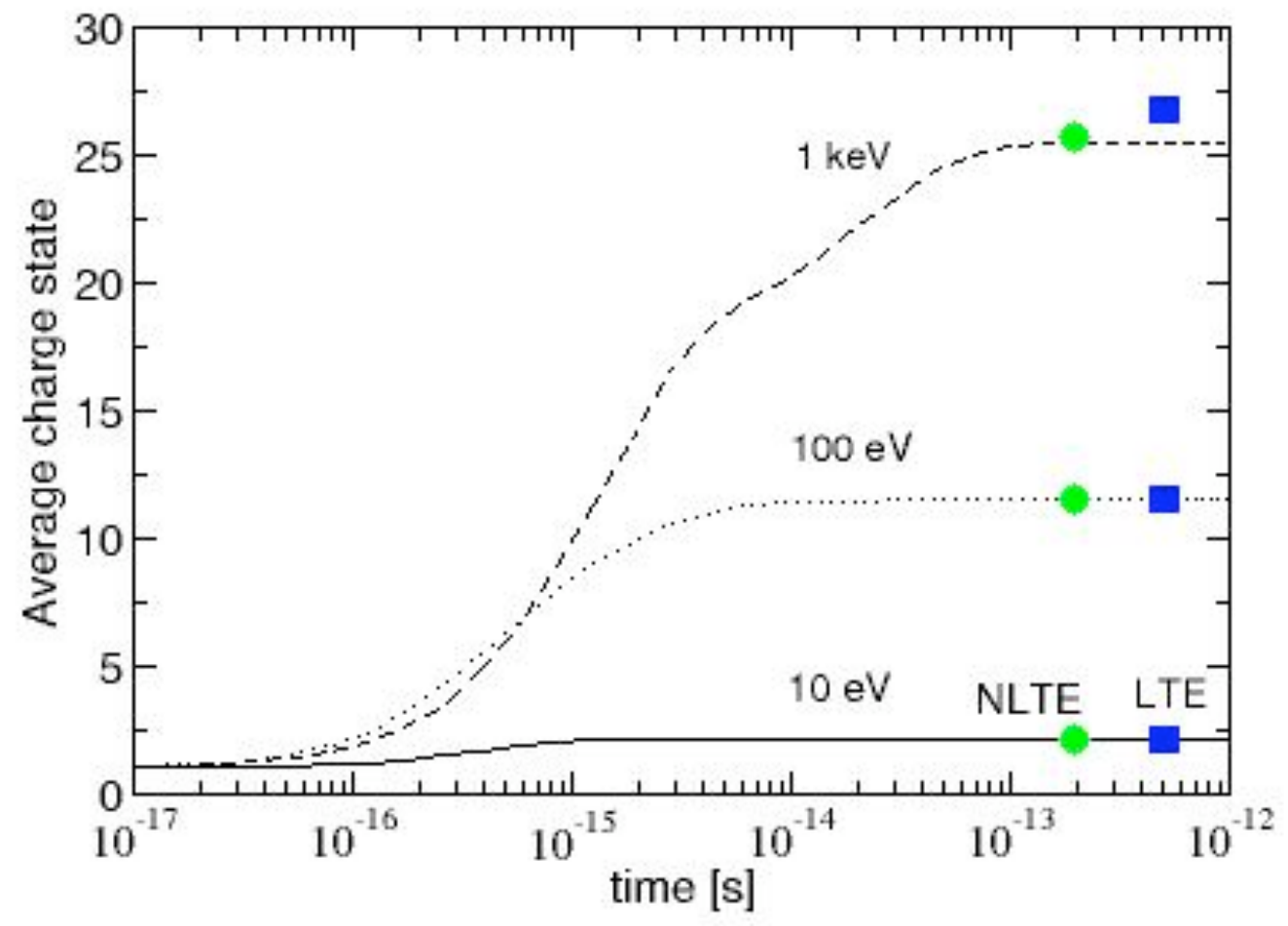




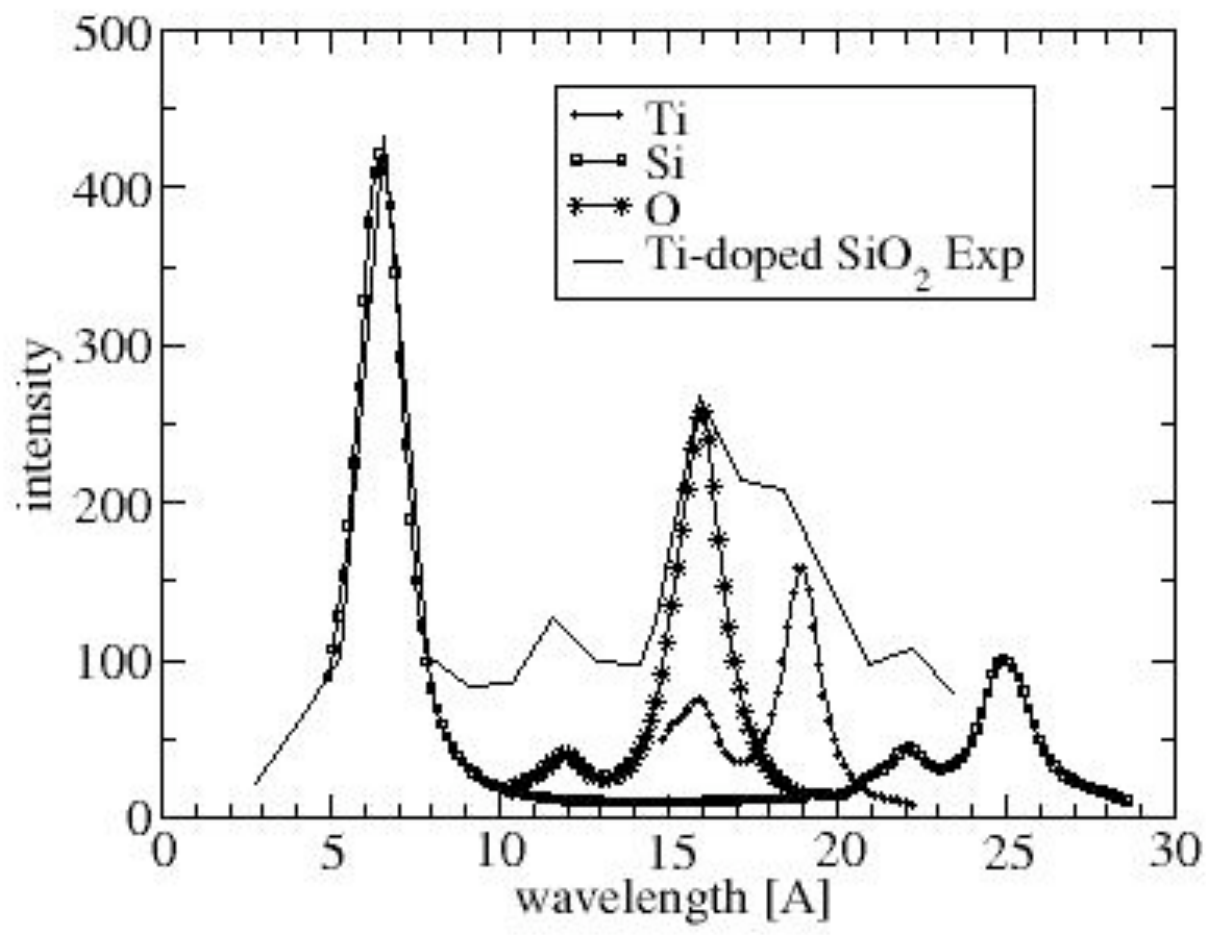




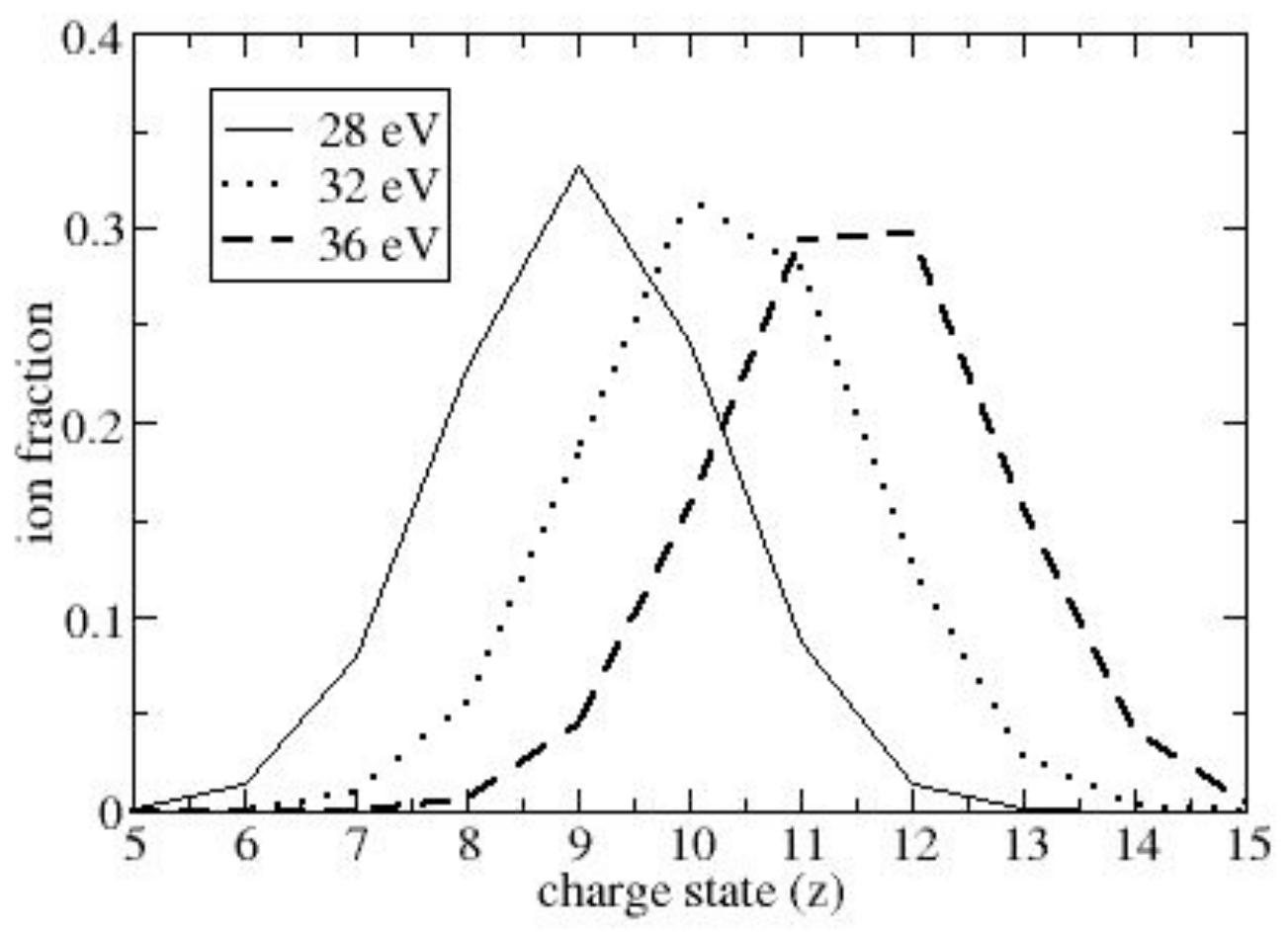




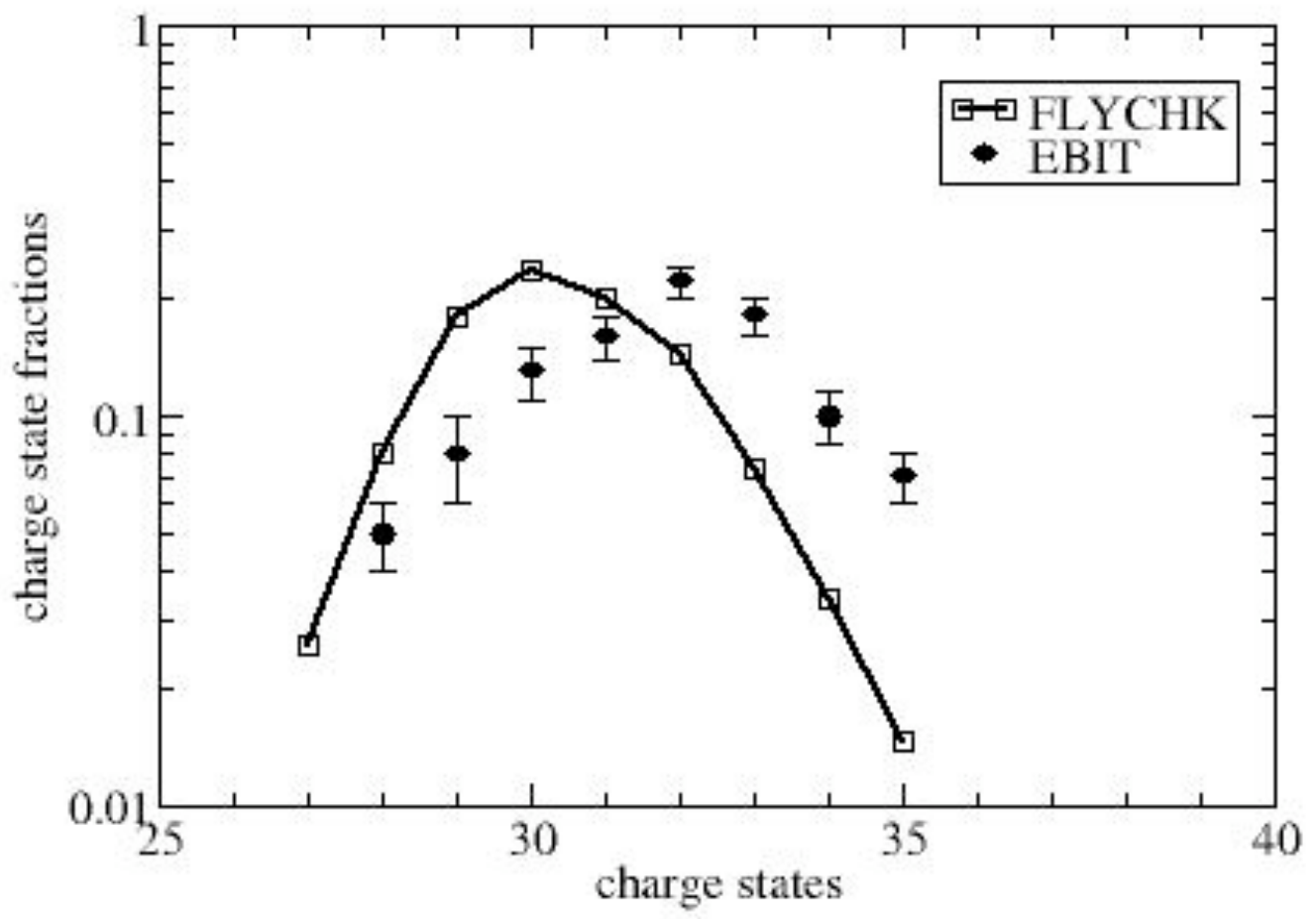

